\title{
MULTI-POINT CONCURRENT TRACKING AND SURVEYING IN CONSTRUCTION FIELD
}

S. Mao ${ }^{1}, *$ M. Lu ${ }^{1}$, X. Shen ${ }^{1}$ and U. Hermann ${ }^{2}$

${ }^{1}$ University of Alberta

Department of Civil and Environmental Engineering

Edmonton, Alberta, Canada

(*Corresponding author: mlu6@ualberta.ca)

${ }^{2}$ PCL Industrial Management Inc.

Edmonton, Alberta, Canada 


\title{
MULTI-POINT CONCURRENT TRACKING AND SURVEYING IN CONSTRUCTION FIELD
}

\begin{abstract}
Total station has been predominantly applied in stationary survey scenarios in construction. With the moving target tracking and point survey automation functions, a robotic total station (RTS) lends itself well to more dynamic applications. Nevertheless, a RTS unit is still unable to track multiple targets simultaneously or to survey a target point when the line-of-sight is temporarily unavailable. In this paper, we propose a methodology to synchronize and automate multiple RTS units intended to address dynamic tracking and surveying applications in the construction field. By using the application programming interface (API) from the manufacturer, we implement the proposed methodology and the prototype system integrates two RTS units in regard to space and time and communicates survey command instructions among RTS units through wireless networks. The two RTS units can be operated to survey the two ends of one swinging sling concurrently in order to retrieve the actual length of the sling within several millimeters accuracy. Besides, in applications where line-of-sight between a target object and one RTS unit cannot be guaranteed all the time, continuous surveying can be materialized by automatically delegating the task to another synchronized RTS unit with clear view of the target object. A field test was conducted to demonstrate the application of the proposed automation approach in which sling length was measured of a rigging framework used for lifting industrial modules. The testing results are presented and achievable accuracy and reliability are discussed. The proposed solution overcomes the limitations of global positioning system (GPS) and laser scanning and holds high potential to provide cost effective solutions to many dynamic field applications.
\end{abstract}

\section{KEYWORDS}

Robotic Total Station, Dynamic Surveying, Automation, Tracking Technology, Rigging.

\section{INTRODUCTION}

Construction is an activity to convert designed objects on the blueprints to the actual objects existing in reality (Bernold, 2002); construction surveying is the key not only to setting out where to build the objects, but also to measuring how accurately the construction process reproduces the design model in the real world (Bernold, 2002). Starting from very basic and simple tools like ruler, survey equipment has gradually evolved. Especially in the last few decades, the advent of new technologies like computer, satellite and laser has taken the development of surveying methods to a new height. By incorporating electronic distance measure instruments (EDM), traditional theodolite can measure distance and angle data in an integrative fashion, significantly simplifying the surveying process (Bernold, 2002). This integration has led to the invention of total station, which has been widely adopted as the common practice of survey. Through technological advances and integration, total station provides the most accurate, stable and reliable means for survey and positioning applications in construction engineering.

However, total station is mostly used in stationary survey settings, in which the target stays still during the survey process and the total station retrieves the static coordinates of the target. This well fits the survey needs of traditional construction methods in two undertakings: (1) land surveying is done prior to the start of construction in order to stake out the site layout; and (2) after construction is completed quantity surveying is generally carried out to certify quality and quantity of finished products. Between the two surveying undertakings, there exists a "blind" period during which the construction process commonly takes place in the field in absence of specialist surveyors. Thus possible errors can occur and accumulate. Nonetheless, more frequent field survey needs means increased workload for the specialist surveying crew while inflating the overhead cost of the project; moreover, how to schedule the construction crew and the survey crew without any unnecessary interruption is a demanding task. In addition, a more critical question is raised: in a complex construction process, how to ensure construction crew is informed of the feedback from the survey crew in time, as such, field construction is not delayed? A better solution 
is called for so as to minimize the "blind" period and improve the quality and efficiency of construction. Undoubtedly, the solution to seamless construction-survey integration lies in the development of technologies to automate real-time high-accuracy survey in the field, and the deployment of the technologies in the field without interfering normal operations by the construction crews. This paper describes a general method to solve these two problems. We propose a methodology framework and develop a prototype to perform high accuracy dynamic survey using a network of synchronized RTS units, as illustrated with a field test-bed.

\section{LITERATURE REVIEW}

As previously stated, we need to build up a coherent system which can retrieve real time positions of dynamic targets. However, total station requires availability of line-of-sight and fails to concurrently track multiple targets. In spite of advanced automation functions for tracking dynamic targets, a single total station can neither track multiple targets at the same time nor function properly when line-of-sight to the target is lost. Emerging survey technologies, including global positioning system (GPS), laser scanner and photogrammetry, attempt to tackle the survey or position challenges in different ways. GPS doesn't need the line-of-sight on the ground and only requires open-sky between the antenna and the satellites (Bernold, 2002). Photogrammetry is based on stereo imaging algebra/geometry. By capturing an image at an exact time point, the photogrammetry can compute the coordinate of each pixel which is covered by two or more images. One additional benefit to snap a "freeze" shot of the target is that photogrammetry can handle dynamic observation easier than other technologies, as all measurements can be taken at the same time moment, which can be readily guaranteed by synchronizing the system clocks of multiple cameras. Laser scanner can profile a massive object in reality, where the laser scanner equipment is placed as the sphere centre. With fast laser reflection, the laser scanning represents the object in a "point cloud" model. Nonetheless, each technology still confers own limitations that potentially undermine its effectiveness in dynamic applications in construction. For instance, GPS cannot be applied to underground circumstances or even any environment with high-rise mental structures in the open sky; in either situation, reliability and accuracy of the GPS results can be significantly downgraded. The limitation for photogrammetry lies mainly in low precision, which is determined by the quality of cameras and the geometry of control points. Laser scanner takes a considerable turnaround time to finish a full cycle of scanning and point cloud data processing (in days), thus, it is more suitable for relatively time-independent, "stationary" as-built modeling.

Based on the comparison of existing methods, we propose a multiple robotic total station networking solution in order to build an automation solution for dynamic construction surveying applications in the field. The two limitations in connection with RTS can be addressed by using a RTS network. Previous efforts of research and application of multiple total station integration are reviewed. Berberan, Machado, \& Batista (2007) studied multiple total station surveying and error propagation based on a curved tunnel construction in Lisbon. The multiple total stations were deployed section by section along the tunnel. Although all the total stations were setup in the same coordinate framework, they worked independently (Berberan et al., 2007). Bao et al. developed a similar robotic total station network, in which all total stations were linked to respective workstation PCs and controlled by a central server PC; however, emphasis of this system was placed on controlling the operation order and work status of total stations; notably, accurate time synchronization and dynamic observation were not addressed (Bao et al., 2006). Jeong et al. built up a mobile surveying system by integrating mobile robots and surveying tools; their system consisted of a surveying robot and two reference points, and whenever the surveying robot moved, it surveyed the references before and after movements and relocated itself using resection (Jeong, Pyo, Iwashita, Hasegawa, \& Kurazume, 2012; Ghilani, 2010). Oloufa et al. (2003) proposed an algorithm to detect construction equipment collision by using GPS. GPS data were collected at a given frequency through wireless connections, so as to calculate the speed vector of the equipment. The collision was described as intersection of moving vectors in the future; finally all the information was displayed in a Web interface (Oloufa, Ikeda, \& Oda, 2003). This system had a similar central server to collect and compute survey data; however, this server only collected data in one direction. In our solution, a server computer provides the brain to coordinate and control the whole system.

This paper presents a new method, which synchronizes multiple robotic total stations into the same time and geospatial frameworks and is able to observe and track dynamic targets. Although iSpace, a similar laser-based solution developed by NIKON Inc. (NIKON METROLOGY, 2013), can perform dynamic observation with multiple transmitters, it is prohibitively expensive and not intended for construction field application, hence has been rarely used in construction projects. 


\section{METHODS}

\section{System Architecture}

To solve the real-time and dynamic surveying problem, our solution builds a total station network by synchronizing multiple total stations both geospatially and temporally. In this system, each component and function is abstracted to be independent of the actual implementation; and for every device in the system, communication can be realized without knowing any detail about the connection method, the communication method, the device protocol or any other physical details. As shown in Figure 1, the system consists of four abstraction layers, each of which provides a unified interface to enable similar operations.

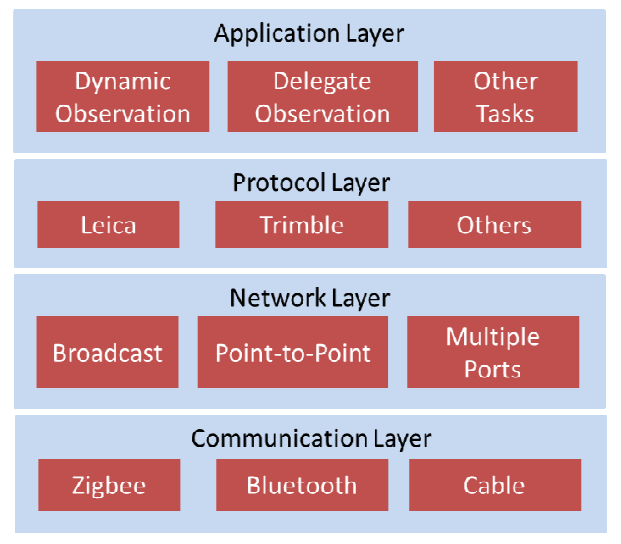

Figure 1 - Architecture of the Control System

The most fundamental layer is the communication layer, which handles different types of connections. By using the communication layer, the program can treat a wired or wireless connection as a duplex port of sending and receiving data. To fulfill this purpose, it is important to handle the latency resulting from applying different networks. In addition, another important task of the communication layer is to control the delay over different connections. Therefore, the time synchronization feature is guaranteed by such delay control.

The second layer is the network layer which wraps different routing methods, for example, ZigBee has broadcast mode, multi-cast mode and peer-to-peer mode. By using the network layer, one device can find and contact other devices without routing details. For example, one total station cannot measure the target because a truck blocks the line-of-sight. With the network layer, this total station reports to the central controller of the target surveying problem. The routing algorithm will then be triggered to assign an alternative total station with this job.

The third layer is protocol layer, which abstracts an ideal robotic total station model and encapsulates the control protocols from different manufacturers. Our system controls the total stations through application programming interface (API), which is a clearly defined set of protocols to manipulate the core functions of the robotic total station. With the API, the controller can perform tasks like searching targets, surveying targets, and detecting errors without manual operations on the device. However, different manufacturers define their own API, and generally, they are incompatible with one another. The protocol layer is developed to abstract the protocols as a manufacturer-neutral service platform, and hides details to perform tasks like rotation, surveying or tracking targets. By using the protocol layer, the system can essentially control different total stations by using the same commands.

The fourth layer is the application layer, which delivers actual surveying tasks and plays as the API of the surveying network. Programmers or operators manipulate the whole system through the application layer, for example, in the sling length measurement application, two total stations are instructed to track each end of the sling, and survey the coordinates of the two ends at the same time. Another example of the application layer is when one total station loses line-of-sight with a target, the delegation of surveying the target to another total station will be initiated; note the application layer encapsulates details of the network layer and the protocol layer.

\section{Total Station Synchronization}


The most critical issue of the concurrent tracking is the coordination of multiple tracking agents (i.e. RTS units in this application) in regards to geospatial data coordination and time coordination. The geospatial coordination is the crucial feature that every surveying procedure must implement, in order to ensure all the data retrieved during the surveying process are represented in a unified coordinate system. And for time coordination, the system should measure and store the latency (the lag in executing commands at the same time) between different devices and networks. For example, a wired network has a lower latency compared to ZigBee networks, while total stations with magnetic motors can locate targets faster than those with normal motors. By figuring out the latency, the system sends requests in a special order with special intervals, ensuring multiple total stations perform survey instruction at the same time.

For traditional traverse surveying, one team or multiple teams start from benchmark control points and ensure all the following surveyed points are recorded or transformed into the common coordinate system. Similarly, in the described multiple points tracking system, the geospatial framework needs to be set up in the same method for obtaining consistent data.

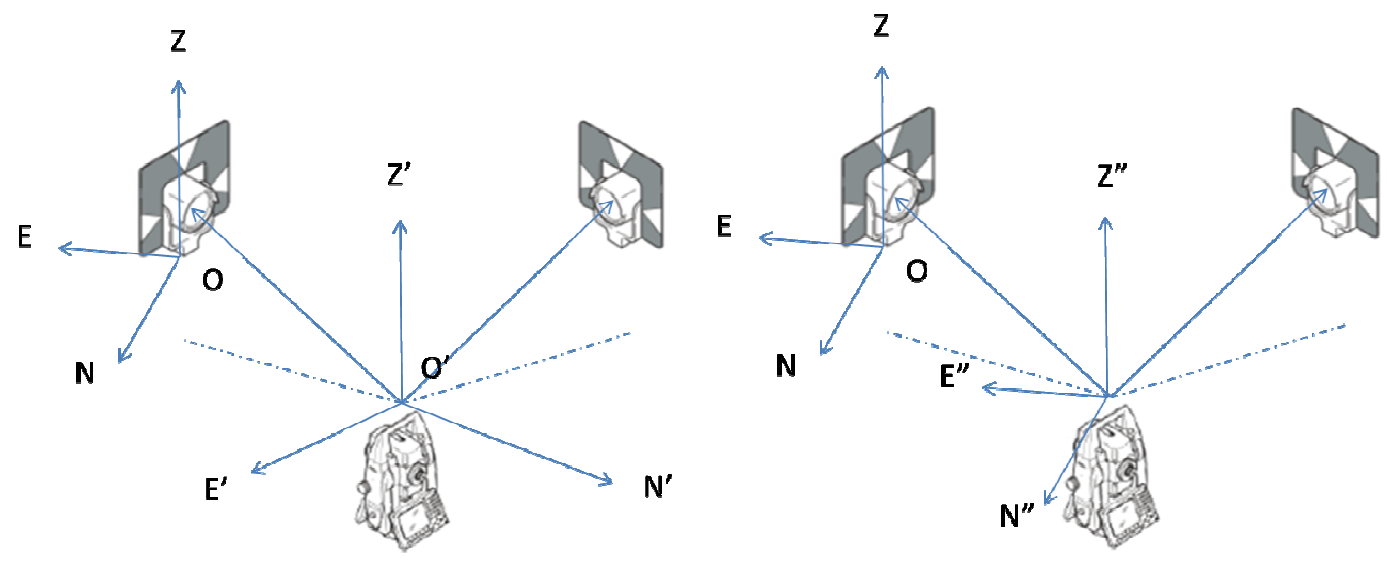

Figure 2 - Resection

During the traverse surveying, every time after the team moves the total station to a new unknown place, a method named resection should be performed before any survey can be conducted. As shown in the left image in Figure 2, two prisms are both set up with known positions in the $O-E N Z$ coordinate framework and a total station originally locates itself within the $O^{\prime}-E^{\prime} N^{\prime} Z^{\prime}$ coordinate system. Resection will be performed to place the total station into the same $O-E N Z$ coordinate framework. During the process, the relative locations between the prisms and total station are the same. Only the coordinate system used by the total station is changed. The total station rotates around the Z' axis, and makes E' axis parallel to $\mathrm{E}$ and N' parallel to $\mathrm{N}$; in addition, total station moves the origin $\mathrm{O}^{\prime}$ to $\mathrm{O}$. As shown in the right image in Figure 2, the new $E^{\prime \prime} N^{\prime \prime} Z$ " are parallel to ENZ respectively, and the origin is point $\mathrm{O}$, therefore, the total station is in the same coordinate system as the reference prisms.

Suppose that the coordinates of total station, left prism and right prism in the $O^{\prime}-E^{\prime} N^{\prime} Z^{\prime}$ coordinate framework are $T^{\prime}, P^{\prime}$ and $Q^{\prime}$, and correspondingly, the coordinates in the $O-E N Z$ coordinate framework are $T, P$ and $Q$. In $O^{\prime}-E^{\prime} N^{\prime} Z^{\prime}$ coordinate framework, the horizontal and vertical angles of left prism and right prism, measured from total station are $H, V, h$ and $v$, respectively. In addition, the slope distances between the total station and the two prisms are $S$ and $D$. The rotation angle that makes the two coordinate systems aligned is assumed to be $\alpha$, and the translation vector that moves one origin to the other is $W$. Therefore, based on all the measured and assumed values, the constraints between the variables can be illustrated as: (Ghilani, 2010)

$$
T^{\prime}+\left[\begin{array}{c}
\cos V \cdot \sin H \cdot S \\
\cos V \cdot \cos H \cdot S \\
\sin V \cdot S
\end{array}\right]=P^{\prime}
$$




$$
\begin{gathered}
T^{\prime}+\left[\begin{array}{c}
\cos v \cdot \sinh \cdot D \\
\cos v \cdot \cosh \cdot D \\
\sin v \cdot D
\end{array}\right]=Q^{\prime} \\
T+\left[\begin{array}{c}
\cos V \cdot \sin (H+\alpha) \cdot S \\
\cos V \cdot \cos (H+\alpha) \cdot S \\
\sin V \cdot S
\end{array}\right]=P \\
T+\left[\begin{array}{c}
\cos v \cdot \sin (h+\alpha) \cdot D \\
\cos v \cdot \cos (h+\alpha) \cdot D \\
\sin v \cdot D \\
T=T^{\prime}+W
\end{array}\right]=Q
\end{gathered}
$$

Given the above equations, the system can compute the unknown $\alpha$ and $W$ (Ghilani, 2010), and produce the transform matrix between the two coordinate systems. According to the structure of the equations, the minimum number of reference points required is two: when only one point is available, the total station cannot perform selfpositioning; meanwhile, if more than two points are available, the equations can be adapted for adjustment algorithm, like the Least Squares Algorithm (Ghilani, 2010). Moreover, the calculation process for $\alpha$ can be simplified based on one observation: $\mathrm{Z}$ and $\mathrm{Z}$ ' axes both align to the direction of gravity, thus to transform one coordinate framework to the other, only a three-dimensional translation vector $W$ and a rotation angle $\alpha$ around $\mathrm{Z}$ axis are needed to be calculated; in another word, the transformation can be treated as rigid body movement. We first compute the vector from $P_{1}$ to $P_{2}$ in two different coordinate systems, then project the two vectors to the EN plane. Thus, the angle between two projected vectors is the rotation angle $\alpha$.

In another word, the whole process of resection can be easily decomposed into two steps: compute the rotation angle $\alpha$, and make each corresponding axis in $O^{\prime \prime}-E^{\prime \prime} N^{\prime \prime} Z$ " and $O-E N Z$ parallel and in the same direction; then compute translation vector $W$, which moves $O "$ to $O$, and makes the two coordinate systems coincide. A numerical example is illustrated here. There are two reference points $P_{1}$ and $P_{2}$ in the same coordinate system, and their coordinates are $P_{1}:\left(\begin{array}{lll}1 & 1 & 1.414\end{array}\right)$ and $P_{2}:\left(\begin{array}{lll}0.433 & 0.75 & 0.5\end{array}\right)$. A total station which is set in another coordinate system surveys the two reference points and the coordinates are $Q_{1}:(2.366-1.366-4.414)$ and $Q_{2}:(1.75-1.433-5.5)$. Then we compute two vectors, $V_{P 2-P 1}:\left(\begin{array}{lll}-0.567 & -0.25 & 0\end{array}\right)$ and $V_{Q 2-Q 1}:\left(\begin{array}{lll}-0.616 & 0.067 & 0\end{array}\right)$, and the $\mathrm{Z}$ values for both vectors are 0 because we only need to calculate the rotation angle in the EN plane. Then we can calculate the angle between $V_{P 2-P 1}$ and $V_{Q 2-Q 1}$, which is $\alpha: 30$ degrees. After the rotation, we can compute the coordinates of $Q_{1}$ and $Q_{2}$ in the $O$ " $-E " N " Z$ " coordinate system, and we name the rotated $Q_{1}$ and $Q_{2}$ as $R_{1}$ and $R_{2}$. The coordinates of $R_{1}$ and $R_{2}$ are $R_{1}:\left(\begin{array}{lll}2 & 0 & -4.414\end{array}\right)$ and $R_{2}:\left(\begin{array}{lll}1.433 & -1.75 & -5.5\end{array}\right)$, by comparing $R_{1}$ and $R_{2}$ to $P_{1}$ and $P_{2}$, we can compute the translation vector $W:\left(\begin{array}{lll}1 & -1 & -5\end{array}\right)$. After having $\alpha$ and $W$, we can transform total station's coordinate system into the reference coordinate system.

Similarly, the whole system can be set up step by step following the above resection methods. Taking Figure 3 for example, there are two reference points and four total stations in the system, and the two references are "Reference 0" and "Reference 1", and the total stations are "Total Station 0" to "Total Station 3" respectively. The coordinates of the two references can be either surveyed by GPS, or surveyed by total station 0 . In the second occasion, we set total station 0 as the origin in the local coordinate system, and the $\mathrm{N}$ axis in this system is pointing from total station 0 to reference point 0 . After setting up the coordinate system, total station 0 surveys both reference 0 and reference 1 , and locates them as $P_{1}$ and $P_{2}$. Then we apply the resection method to each total station: every total station surveys the two references and gets the coordinates $Q_{1}^{i}$ and $Q_{2}^{i}$, and following the numeric example, we can compute $\alpha^{i}$ and $W^{i}$ for each total station, and transform the position of each total station into the reference coordinate system. 


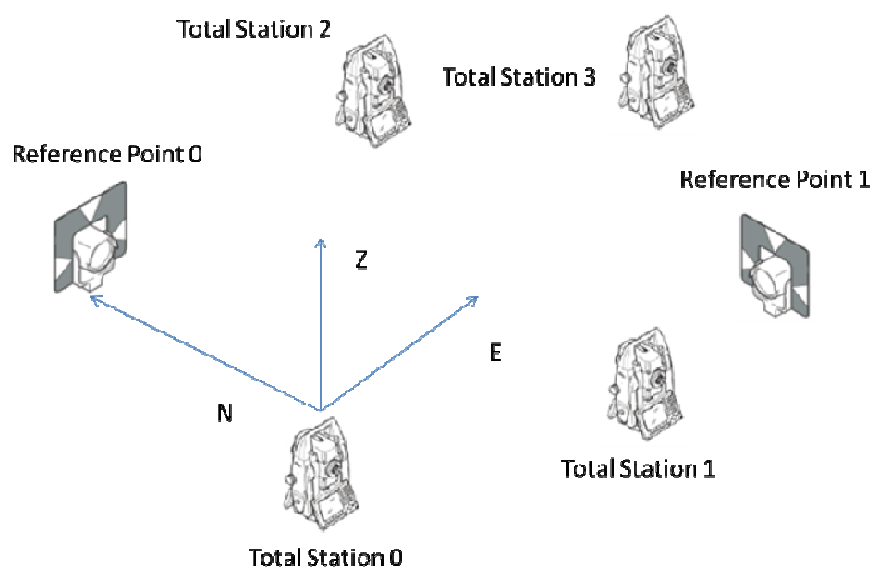

Figure 3 - Geospatial Coordination of the Whole System

In addition, the time coordination is another fundamental setup of the system. This actually defines the main difference between this new multi-RTS system and other similar systems. In a traditional surveying system, when different survey teams are sent out to measure one or several targets, basically they have two options to carry out the measurements on the same time point. One option is to coordinate the clocks ahead of time, and schedule a time to take measurements; the other option is to communicate through low-latency radio or phone and choose a coordinator, and take measurements when the coordinator gives the order. The later method is limited by the latency of the communication method and also affected by the reacting time between hearing and performing the order. The previous one is mainly used to survey static objects. Besides the above two options, another practical method can also approximately ensure concurrency: measuring the targets on a given frequency from all the devices and keep records for a definitive time span. The accuracy of this method highly depends on the choice of frequency and the synchronization of all involved system clocks. In contrast, the strategy for time coordination used in the proposed system can be taken as a combination of the first two methods. The computer plays the role of a human coordinator while the wired or wireless connections between the computer and the robotic total stations serve as the phone or radio. In this system, the central computer commands all of the related robotic total stations to survey dynamic target points at the same time. Then, the computer receives all the time-stamped results via wireless data communication networks. Generally, the communication method is chosen to be identical across all the devices, thus the latency between the computer and each surveying device does not vary significantly.

\section{EXPERIMENTS}

The synchronized surveying network was field-tested in a rigging-lifting test bed. The rigging frame has two sides, and each side is illustrated in Figure 4; on each side, a beam is connected with three pairs of slings. All the slings has one end fixed on the beam and the other end on a Y-Plate above, which will be hooked by the crane at point $\mathrm{O}$. Structurally, controlling the lengths of the twelve slings (two sides) is critical to keep the frame in balance during a heavy lift job. The testing is designed to measure slings' lengths when the rigging frame is being lifted by a crane. When the frame is loaded with tons of weights, the slings will elongate and the actual sling lengths will be different from the designed values, which may results in imbalance and potential safety hazards.

The rigging frame swings in the air under the gravity and the wind load when it is lifted. Different components of the whole structure will swing in different patterns. To solve this dynamic surveying problem, we implemented the synchronized surveying network. As shown in the left image of Figure 4, two robotic total stations were setup on the same side of the frame. RTS 1 was set as the origin of the local coordinate system, and the two reference prisms were located; by applying coordinate synchronization, RTS 2 was set within the same coordinate framework; then two prisms were mounted on the centres of the two ends of a sling. After lifting the frame, length surveying was done sling by sling, and RTS 1 was assigned to track the prism on the beam and RTS 2 was assigned to track the prism on the Y-plate. The sample of surveyed data are shown in Table 1, each pair is surveyed by the total stations at the same time. In the table, "Time 1" is the time when a button is pressed on the API to order a survey, and "Time 2" is the time when the system receives the responses from total stations. Note in the current 
robotic total station API protocol the exact times when total station receives an order and performs the survey are not published, but we can obtain the time stamps when the system receives the responses. Two factors affect the performance of the proposed method: (1) the tracking and locking time the total station spends in surveying a target and (2) the latency in data communication. As for the tracking and locking functions, if the target is moving fast in one direction, it is easier for a total station to predict and lock its position. However, when the target swings in a circle, it is more difficult and takes longer for total station to track. This is accounts for why some measurements have larger differences in Time 2 (note they are supposed to be identical.) Moreover, due to the large size of the rigging, the looking angle is far from the perpendicular angle, thus making it difficult for the total stations to survey some targets.

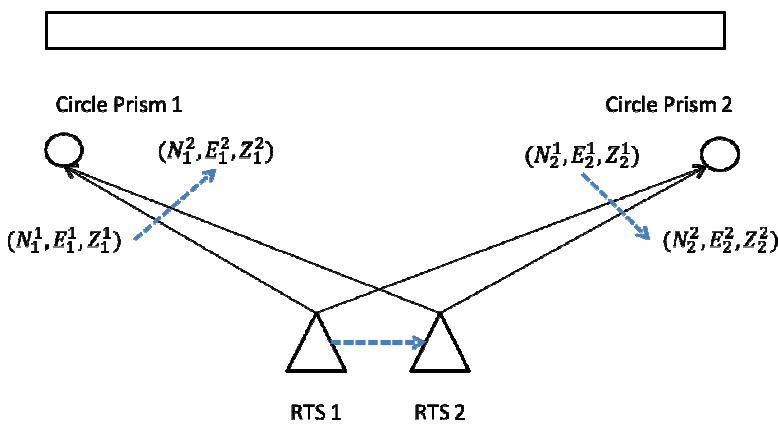

Top view

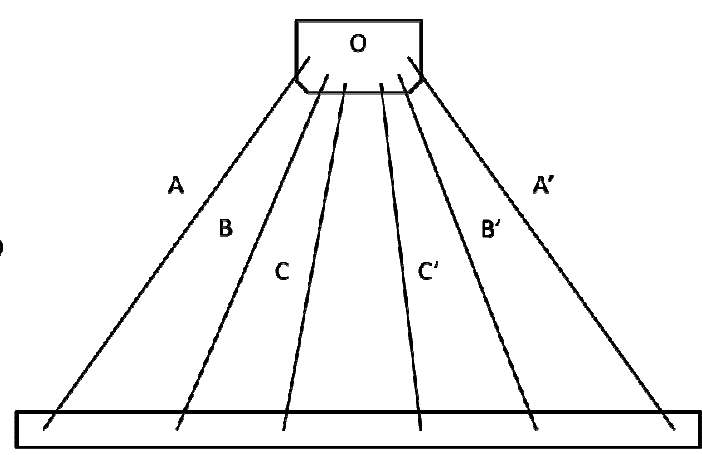

Section View

Figure 4 - Site Setup

Table 1 - Sample of surveyed data, one measurement for each sling

\begin{tabular}{|c|c|c|c|c|c|c|c|}
\hline Slings ID & Ends & $\mathrm{E} / \mathrm{X}(\mathrm{m})$ & $\mathrm{N} / \mathrm{Y}(\mathrm{m})$ & $\mathrm{Z}(\mathrm{m})$ & Length (m) & Time 1 & Time 2 \\
\hline \multirow{2}{*}{$\mathrm{B}^{\prime}$} & Lower & 15.331 & 38.219 & -0.568 & \multirow{2}{*}{18.205} & $2: 45: 45$ PM & 2:45:49 PM \\
\hline & Upper & 23.268 & 37.058 & 15.775 & & $2: 45: 45$ PM & $2: 45: 49 \mathrm{PM}$ \\
\hline \multirow{2}{*}{ B } & Lower & 32.760 & 35.738 & -0.383 & \multirow{2}{*}{18.230} & 2:50:01 PM & 2:50:09 PM \\
\hline & Upper & 24.438 & 36.898 & 15.795 & & 2:50:01 PM & $2: 50: 11 \mathrm{PM}$ \\
\hline \multirow{2}{*}{$\mathrm{A}^{\prime}$} & Lower & 9.550 & 39.206 & -0.633 & \multirow{2}{*}{21.507} & $2: 54: 16$ PM & $2: 54: 26 \mathrm{PM}$ \\
\hline & Upper & 23.027 & 37.145 & 16.000 & & $2: 54: 16$ PM & $2: 54: 26$ PM \\
\hline \multirow{2}{*}{ A } & Lower & 37.447 & 32.546 & -0.309 & \multirow{2}{*}{21.503} & $2: 58: 49$ PM & 2:58:57 PM \\
\hline & Upper & 24.136 & 36.873 & 16.015 & & 2:58:49 PM & 2:58:57 PM \\
\hline \multirow{2}{*}{$\mathrm{C}^{\prime}$} & Lower & 20.743 & 37.967 & -0.518 & \multirow{2}{*}{16.279} & $3: 00: 45$ PM & 3:00:52 PM \\
\hline & Upper & 23.151 & 37.184 & 15.563 & & 3:00:45 PM & $3: 00: 53 \mathrm{PM}$ \\
\hline \multirow{2}{*}{$\mathrm{C}$} & Lower & 26.344 & 36.187 & -0.458 & \multirow{2}{*}{16.280} & 3:02:05 PM & $3: 02: 12 \mathrm{PM}$ \\
\hline & Upper & 23.597 & 37.035 & 15.566 & & 3:02:05 PM & 3:02:13 PM \\
\hline
\end{tabular}

The time duration to survey all six slings took about 17 minutes and 17 seconds. As the frame is a dynamic structure, the length of the swinging slings may change when they are loaded. Therefore when the lag is relatively large, it is invalid to assume that the two total stations survey the targets at the same time. In our field test, every sling was measured at least three times, and if some surveys failed, more measurements would be collected because these slings swung considerably and the total station had difficulty in locking them. Finally, 20 measurements were collected, and they were divided into six groups, each group standing for one sling, namely: B', B, A', A, C' and C. According to Figure 5, 70\% of the lags are less than 1 second, thus these data were taken as valid, and we then computed the sling length by averaging all valid data in a sling group. Note, if there were no measurements in a group with Time 2 lags less than 1 second, we chose the least values available, and treat them as the approximation of the sling length. As shown in Table 1, the worst case occurs in group B, in which no lags on Time 2 were less than 1 second, thus we took the length computed from the records with 2 seconds lag. 


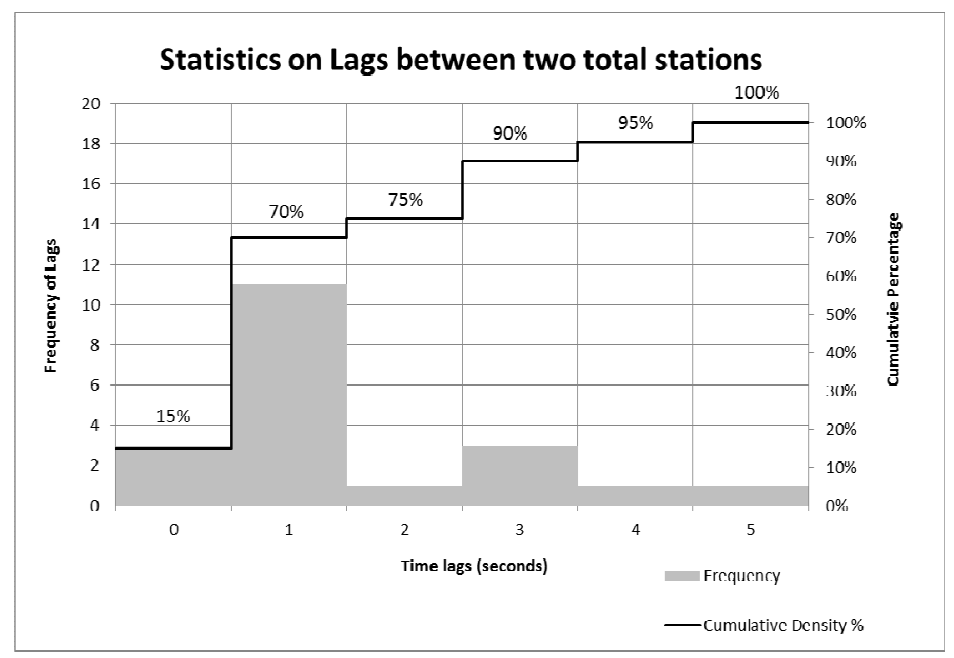

Figure 5 - Statistics on time lags between the two total stations

\section{CONCLUSIONS}

This paper presents a method to survey multiple dynamic targets in real-time in construction fields. This method realizes space integration and time synchronization to extend the previous methods for applying multiple total stations. A synchronized total station network prototype was built, in which every total station was controlled to survey dynamic targets at the same time, therefore solving problems like sling length measurement in the field. To implement the system, a software platform was designed which abstracts details like communication methods and total station protocols, and this platform makes it very easy to implement synchronization and control of multiple RTS units in the system. In addition, the resection algorithm is chosen as the geospatial synchronization method and explained in detail. Time synchronization is designed to reduce the latency between different components, and it is handled by the software platform in a unified method and by wireless networks. A riggingframe-lifting case study was conducted as a proof of the synchronization method, and the results are analyzed and presented.

There are two immediate improvements to be implemented in the future. First, a more elaborate latency estimation algorithm should be designed. A main difficulty we faced during the test was to lock the swinging targets; it was difficult for total station to predict the moving pattern and sometimes took longer time than usual. The new algorithm should be more adaptive and be able to predict the locking time of total station more precisely, thus allowing adjustment on the latency for the next survey. As such, the system can be more accurate in dynamic applications. Second, the synchronized network may integrate with other kinds of survey technologies (photogrammetry/GPS) in order to further improve the versatility, accuracy and reliability.

\section{ACKNOWLEDGMENTS}

The writers acknowledge PCL Industrial Management Inc. for assistance support during field testing. The writers also thank Ming-fung Siu, Hexu Liu and Xiaodong Wu for facilitating field testing.

\section{REFERENCES}

Bao, H., Zhao, D., Fu, Z., Zhu, J., Sun, F., \& Wei, J. (2006). Application of Network TPS Intelligent Monitoring System in Subway Structure Deformation Monitoring. In Proceedings of 3rd IAG / 12th FIG Symposium. Presented at the 3rd IAG Symposium on Geodesy for Geotechnical and Structural Engineering / 12th FIG Symposium on Deformation Measurement, Baden, Austria. 
Berberan, A., Machado, M., \& Batista, S. (2007). Automatic multi total station monitoring of a tunnel. Survey Review, 39(305), 203-211(9). doi:http://dx.doi.org/10.1179/003962607X165177

Bernold, L. (2002). Spatial Integration in Construction. Journal of Construction Engineering and Management, 128(5), 400-408. doi:10.1061/(ASCE)0733-9364(2002)128:5(400)

Ghilani, C. D. (2010). Adjustment Computations: Spatial Data Analysis (5th ed.). Wiley.

Jeong, Y., Pyo, Y., Iwashita, Y., Hasegawa, T., \& Kurazume, R. (2012). High-precision three-dimensional laser measurement system by cooperative multiple mobile robots. In 2012 IEEE/SICE International Symposium on System Integration (SII) (pp. 198 -205). Presented at the 2012 IEEE/SICE International Symposium on System Integration (SII). doi:10.1109/SII.2012.6427377

NIKON METROLOGY. (2013). Brochue of iSpace: Large Volume Metrology, Tracking and Positioning. Retrieved from http://www.nikonmetrology.com

Oloufa, A. A., Ikeda, M., \& Oda, H. (2003). Situational awareness of construction equipment using GPS, wireless and web technologies. Automation in Construction, 12(6), 737-748. doi:10.1016/S0926-5805(03)00057-8 